\title{
Bis(2,3-dibromo-4,5-dihydroxybenzyl) Ether, a Marine Algae Derived Bromophenol, Inhibits the Growth of Botrytis cinerea and Interacts with DNA Molecules
}

\author{
Ming Liu ${ }^{1}$, Genzhu Wang ${ }^{1}$, Lin Xiao ${ }^{2, *}$, Xuanli Xu ${ }^{3}$, Xiaohui Liu ${ }^{3}$, Pingxiang $\mathrm{Xu}^{3}$ and \\ Xiukun Lin $^{3, *}$
}

1 Key Laboratory of Marine Drugs, Ministry of Education, School of Medicine and Pharmacy, Ocean University of China, Qingdao 266003, China; E-Mails: 1mouc@ hotmail.com (M.L.); 15133634098@163.com (G.W.)

2 College of Chemistry and Pharmaceutical Sciences, Qingdao Agricultural University, Qingdao 266109, China

3 Department of Pharmacology, Capital Medical University, Beijing 100069, China; E-Mails: xh152@msn.com (X.X.); xiukunlin@126.com (X.L.); xupingxiang@163.com (P.X.)

* Authors to whom correspondence should be addressed; E-Mails: xiaolin_qd@ @au.edu.cn (L.X.); linxiukun@qdio.ac.cn (X.L.); Tel.: +86-532-88030523 (L.X.); Fax: +86-532-86080895 (L.X.); Tel./Fax:+86-1083911678 (X.L.).

Received: 23 January 2014; in revised form: 12 May 2014 / Accepted: 13 May 2014 /

Published: 27 June 2014

\begin{abstract}
Bis(2,3-dibromo-4,5-dihydroxybenzyl) ether (BDDE) is a bromophenol isolated from marine algae. Previous reports have shown that BDDE possesses cytotoxic and antibacterial activity. In the present study, we demonstrate that BDDE displays broad-spectrum antifungal activities, especially on Botrytis cinerea. BDDE inhibits the growth of $B$. cinerea cultured on a solid medium of potato dextrose agar (PDA) as well as on the potato dextrose broth (PDB) medium. Moreover, BDDE decreases the incidence of fruit decay and severity of strawberries infected with $B$. cinerea. Further studies have revealed that BDDE decreases the germination rate and inhibits the mycelial growth of $B$. cinerea. The inhibition mechanisms are related to the disruption of the cell membrane integrity in B. cinerea spores and newly formed germ tubes. This study also suggests that BDDE possibly interacts with DNA via intercalation and minor groove binding. The studies provide evidence that BDDE has potential application in the control of gray mold after fruit harvest and the compound could serve as a candidate or lead template for rational drug design and for the development of antifungal agents.
\end{abstract}


Keywords: bis(2,3-dibromo-4,5-dihydroxybenzyl) ether; Botrytis cinerea; antifungal; DNA interaction

\section{Introduction}

Phytopathogenic fungi, one type of major parasitic organisms, constitute a main threat to plants, and usually induce serious diseases and yield losses in crops [1]. Currently, infection by phytopathogenic fungi on plants, such as gray mold, is generally treated with synthetic fungicides to control susceptible pathogens [2]. Although the synthetic fungicides are effective and used widely, continuous application of these fungicides has resulted in loss of biological control and has led to drug resistance and environmental problems [3]. Moreover, synthetic fungicides often induce concern in food safety. To overcome these problems, continuous efforts to find safer, more effective control options with minimal risk to human health and the environment is urgent.

Marine bromophenols, usually existing in marine sponges and algae, have attracted much attention in the field of antimicrobial agents [4]. Accumulated evidence indicates that marine bromophenols possess promising antibacterial activity [5-7] and antiviral activities [8,9]. For example, a synthesized bromophenol compound, 2,4,6,2',4',6'-hexabromodiorcinol, shows potent antibacterial activity against several pathogenic bacteria with MIC values ranging from 0.556 to $1.11 \mu \mathrm{M}$ [7]. In addition, symphyocladin G, a new bromophenol adduct derived from marine red alga Symphyocladia latiuscula, is found to possess antifungal activity against Candida albicans [10]. Several bromophenols isolated from red alga Odonthalia corymbifera have been reported to be promising candidates for antifungal agents in crop protection. These bromophenols could inhibit the pathogenicity of fungus Magnaporthe grisea and reduce the appressorium formation on rice plants [11].

Bis(2,3-dibromo-4,5-dihydroxybenzyl) ether (BDDE, Figure 1A), isolated from the marine algae Leathesia nana, Rhodomela confervoides, and Rhodomela confervoides, possesses a variety of bioactivities, such as cytotoxicity to cancer cells [12,13], inhibition of protein tyrosine phosphatase 1B [14], and $\alpha$-gulcosidase [15-18]. BDDE also exhibits antibacterial activity against several strains of Gram positive and Gram negative bacteria [6]. In this study, we demonstrate that BDDE displays antifungal activities on several strains of fungal pathogens, and has the potential to control gray mold in fruit caused by Botrytis cinerea. We find that BDDE inhibits the spore germination and germ tube elongation of $B$. cinerea, and the mechanisms are related to the disruption of cell membranes in $B$. cinerea and the interaction with DNA.

Figure 1. Chemical structure of bis(2,3-dibromo-4,5-dihydroxybenzyl) ether (BDDE).<smiles>Oc1cc(COCc2cc(O)c(O)c(Br)c2Br)c(Br)c(Br)c1O</smiles> 


\section{Results}

\subsection{BDDE Inhibits the Mycelial Growth of Fungal Pathogens}

To evaluate the antifungal activities of BDDE in vitro, we examined its inhibition on mycelial growth to seven fungal pathogens. As shown in Table 1 and Figure 2A, BDDE (100 $\mu \mathrm{g} / \mathrm{mL})$ displayed broad and potent inhibition on the mycelial growth of B. cinerea (a), Valsa mali (b), Fusarium graminearum (c), Coniothyrium diplodiella (d), and Colletotrichum gloeosporioides (e), but no inhibition on Alternaria mali Roberts and Alternaria porri (Table 1). Among these pathogens, B. cinerea was relatively more sensitive to BDDE with an inhibition rate of about $83.3 \%$ (Table 1). BDDE caused obvious decreases in the colony expansion cultured on PDA medium plate (Figure 2Aa). In addition, BDDE could also inhibit the mycelial growth of $B$. cinerea in PDB medium (Figure 2B). Therefore, B. cinerea was selected for further analysis.

Figure 2. BDDE inhibits the mycelial growth of fungal pathogens. (A) The inhibitory effect of BDDE on the mycelial growth of fungal pathogens. The fungal pathogens including B. Cinerea (a); Valsa mali (b); Fusarium graminearum (c); Coniothyrium diplodiella (d); Colletotrichum gloeosporioides (e) were cultured on potato dextrose agar (PDA) medium and treated with BDDE for 4, 2, 3, 2, 3 days, respectively. Three replicates were performed for each fungus; (B) BDDE inhibits the mycelial growth of $B$. cinerea cultured in potato dextrose broth (PDB) liquid medium. Fungal spores were pre-germinated in PDB medium for $24 \mathrm{~h}$. Then the spores with germ tube were incubated for another $24 \mathrm{~h}$ in the absence (a) and presence (b) of $100 \mu \mathrm{g} / \mathrm{mL}$ BDDE.

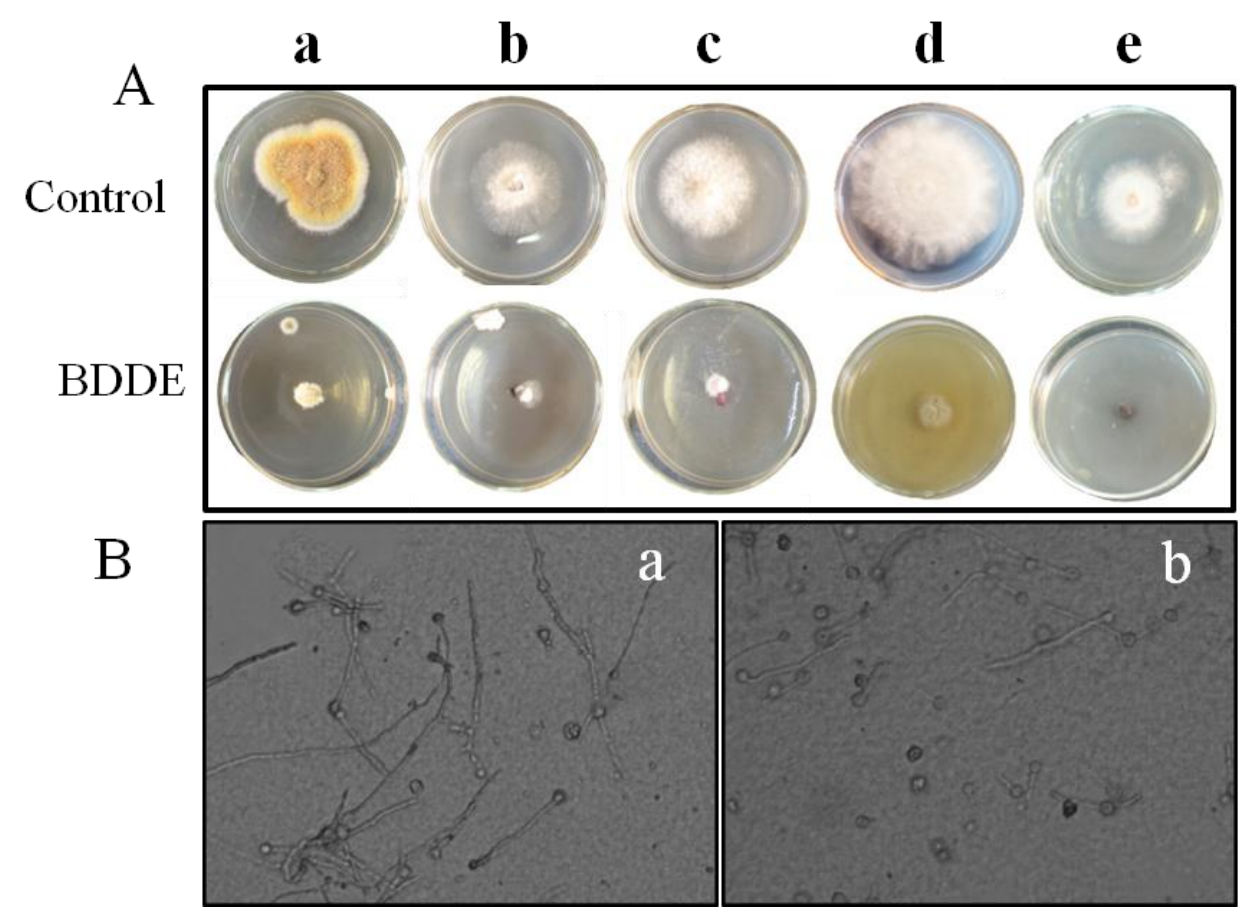


Table 1. Antifungal activities of BDDE against seven fungal pathogens on PDA medium plate containing $100 \mu \mathrm{g} / \mathrm{mL}$ BDDE. Three replicates were used for each fungus.

\begin{tabular}{cc}
\hline Pathogenic Fungi & Inhibition Rate (\%) \\
\hline Botrytis cinerea & $83.3 \pm 6.8$ \\
Valsa mali & $80.0 \pm 7.2$ \\
Fusarium graminearum & $77.1 \pm 5.3$ \\
Coniothyrium diplodiella & $75.0 \pm 8.5$ \\
Colletotrichum gloeosporioides & $67.7 \pm 5.9$ \\
Alternaria mali Roberts & 0 \\
Alternaria porri & 0 \\
\hline
\end{tabular}

\subsection{BDDE Inhibits the Growth of Gray Mold on Strawberries}

To further confirm the antifungal activities of BDDE, fruit decay tests were carried out on freshly harvested strawberries. As shown in Figure 3A, the fruits infected with $B$. cinerea for 5 days showed serious decay in the control group. However, the formation of gray mold was delayed and the decay incidence decreased to $83 \%, 57 \%$, and $39 \%$ when treated with BDDE at a concentration of 25, 50, $100 \mu \mathrm{g} / \mathrm{mL}$, respectively (Figure 3B). These results confirmed that BDDE could inhibit the formation of gray mold on strawberries induced by $B$. cinerea.

Figure 3. Inhibitory effect of BDDE on strawberry gray mold caused by $B$. cinerea. (A) Representative pictures of strawberries treated with $(100 \mu \mathrm{g} / \mathrm{mL})$ or without BDDE for 5 days at $23{ }^{\circ} \mathrm{C}$; (B) Histogram shows the decay incidence in the absence or presence of BDDE. The experiment was repeated three times. Values are expressed as means \pm SD. $* p<0.05$, ** $p<0.01$ versus control indicates significant difference according to Student's $t$-test.
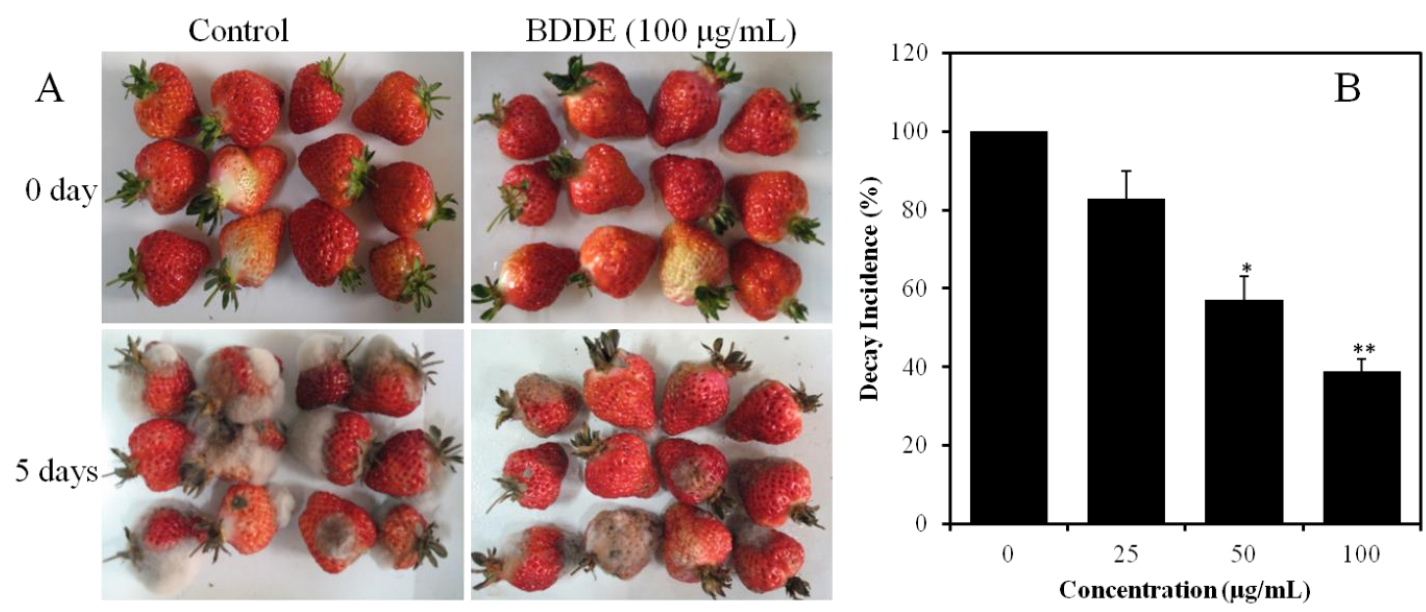

\subsection{BDDE Inhibits Spore Germination and Germ Tube Elongation of B. cinerea}

The effect of BDDE on spore germination and germ tube elongation in PDB medium was investigated. As shown in Figure 4A, the spore germination of $B$. cinerea was significantly inhibited by BDDE in a concentration dependent manner. The germination rate was $87 \%$ in the control group. However, the rate was decreased significantly when the spores were treated with BDDE; the 
germination rate decreased to $74 \%, 45 \%, 39 \%$, and $6 \%$, when treated the spores with $12.5,25,50$, and $100 \mu \mathrm{g} / \mathrm{mL}$ BDDE, respectively (Figure 4B). The $\mathrm{IC}_{50}$ value of BDDE on $B$. cinerea germination is about $31 \mu \mathrm{g} / \mathrm{mL}$. In addition, BDDE also suppressed the elongation of germ tube. Germ tube elongation decreased with the increasing concentration of BDDE, and was almost completely inhibited by BDDE at $100 \mu \mathrm{g} / \mathrm{mL}$ (Figure 4A). These results indicated that both spore germination and germ tube elongation were inhibited by BDDE.

Figure 4. Effect of BDDE on spore germination and germ tube elongation of B. cinerea in PDB. (A) Spores were treated without (a) or with BDDE at a concentration of 25 (b), 50 (c), and $100 \mu \mathrm{g} / \mathrm{mL}$ (d). The germination rate and germ tube elongation were observed using a microscope; (B) Histogram shows the germination inhibition rate in the absence or presence of BDDE. Values are expressed as means \pm SD. $* p<0.05$, ** $p<0.01$ versus control indicates significant difference according to Student's $t$-test.
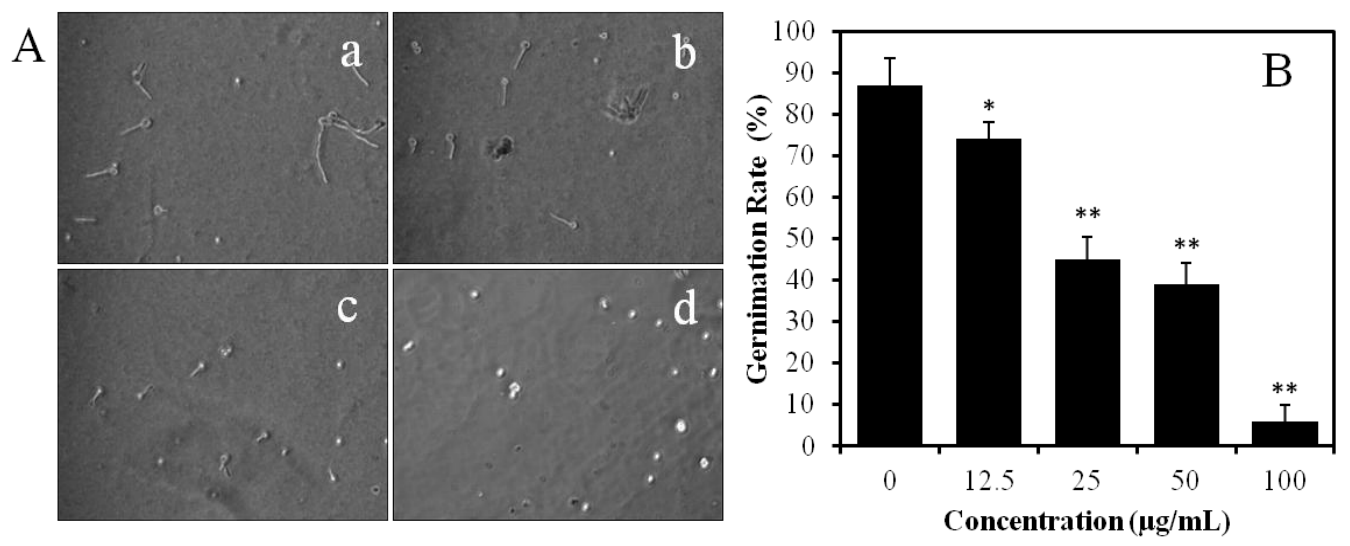

\subsection{BDDE Destroys the Membrane Integrity of B. cinerea}

In order to illustrate the mechanisms underlying the BDDE inhibition against $B$. cinerea, we first detected the membrane integrity of $B$. cinerea using the propidium iodide (PI) staining assay. The PI stained cells from more than 100 spores were counted under a fluorescence microscope. As shown in Table 2, compared with the control group, more spores were stained by PI and the percentage of stained cells increased in a concentration dependent manner; in the absence of BDDE, the percentage of PI stained spores was only 5.6\%. However, it increased to $11 \%, 16 \%$, and $23 \%$ when treated with BDDE at a concentration of 25,50 and $100 \mu \mathrm{g} / \mathrm{mL}$, respectively. These results suggested that BDDE enhanced the membrane permeabilization of $B$. cinerea.

Table 2. Number of total spores and PI stained spores untreated or treated with 25, 50, and $100 \mu \mathrm{g} / \mathrm{mL}$ of BDDE for $24 \mathrm{~h}$, respectively.

\begin{tabular}{cccc}
\hline $\begin{array}{c}\text { BDDE } \\
(\boldsymbol{\mu g} / \mathbf{m L})\end{array}$ & $\begin{array}{c}\text { Total Number } \\
\text { of Spores }\end{array}$ & $\begin{array}{c}\text { Number of PI } \\
\text { Stained Spores }\end{array}$ & $\begin{array}{c}\text { Percentage of PI } \\
\text { Stained Spores }(\%)\end{array}$ \\
\hline 0 & 123 & 7 & 5.6 \\
25 & 116 & 12 & 11 \\
50 & 106 & 17 & 16 \\
100 & 108 & 25 & 23 \\
\hline
\end{tabular}




\subsection{BDDE Interacts with DNA}

It is well established that many antifungal agents can interact with DNA molecules [19]. To investigate if BDDE could interact with DNA, a series of spectroscopic analyses was performed using calf thymus DNA (ctDNA), which is widely used to study the interaction between small molecules and DNA. As shown in Figure 5A, the UV-VIS spectrum of ctDNA displayed an obvious absorption at $260 \mathrm{~nm}$, while BDDE alone only present a relatively low absorption. However, a significant suppression was found when adding BDDE to the ctDNA solution and a red shift of the maximum peak was found, indicating that an interaction happens between BDDE and ctDNA. DAPI can interact with DNA molecules by binding in the minor groove and the major groove [20]. To further confirm the interaction between DNA and BDDE, a DAPI displacement fluorescence assay was employed. The results showed that, in the absence of the BDDE, the peak fluorescent intensity of DNA and DAPI was $265 \pm 16$. However, the intensity decreased to $235 \pm 14$ and $222 \pm 13$, when treated with 15 and $30 \mu \mathrm{M}$ BDDE, respectively. As shown in Figure 5B, the representative fluorescence emission spectra showed that a significant reduction in fluorescence was observed upon treatment with BDDE. The results suggested that BDDE displaced the DAPI from DNA, and there was an interaction between BDDE and DNA.

Figure 5. BDDE interacts with calf thymus DNA (ctDNA). (A) BDDE changes the absorption spectrum of ctDNA. The UV absorption spectra of ctDNA $(50 \mu \mathrm{M})$ at $260 \mathrm{~nm}$ were analyzed in the presence $(100 \mu \mathrm{M})$ and absence of BDDE using a Beckman DU 650 UV-VIS spectrophotometer (Kleve, Germany); (B) DAPI is displaced by BDDE as determined by fluorescence emission spectra. ctDNA $(50 \mu \mathrm{M})$ was incubated with DAPI $(1.5 \mu \mathrm{M})$ or concentrations of $\operatorname{BDDE}(15,30 \mu \mathrm{M})$ for $30 \mathrm{~min}$ at $37{ }^{\circ} \mathrm{C}$, respectively. Fluorescence emission spectra $\left(\lambda_{\max }=488 \mathrm{~nm}, \lambda_{\operatorname{exc}}=340 \mathrm{~nm}\right)$ were determined using a Hitachi F-4500 fluorescence spectrophotometer (Tokyo, Japan). The experiment was repeated more than three times.

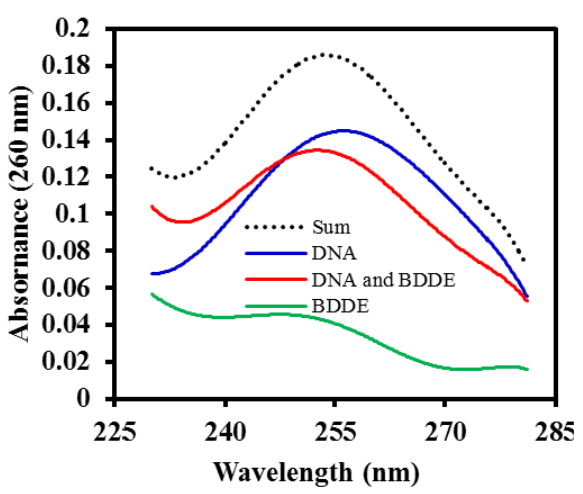

(A)

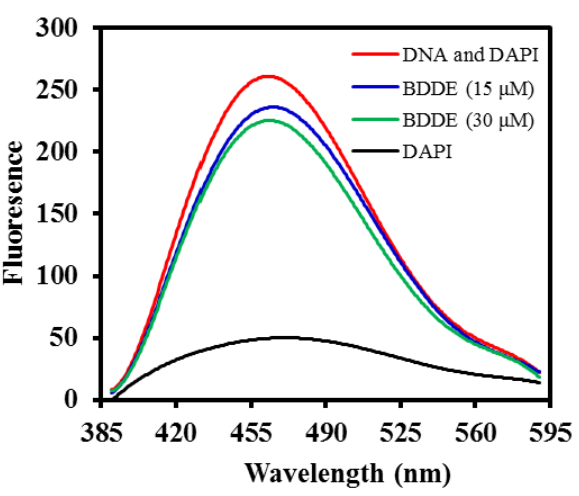

(B)

\subsection{BDDE Intercalates ctDNA and Binds with the Minor Groove of ctDNA}

Acridine orange (AO), a DNA probe agent, can intercalate into DNA molecules [21], and Hoechst33258 is a DNA binding agent, which can interact with the minor groove of DNA [22]. To further study the interaction between BDDE and DNA, displacement experiments were performed. 
The results showed that both AO and Hoechst33258 could be displaced from ctDNA (Figure 6A,B), indicating that the interaction between BDDE and DNA includes both intercalation mode and minor groove binding mode. The result was consistent with our previous study in that BDDE molecule fitted well into the minor groove of the DNA fragment consisting of sequential A-T base pairs [13].

Analysis of the CD spectrum also confirmed the interaction; a distinct change was found in the UV region of the CD spectrum of ctDNA when treated with BDDE, and there was a significant decrease in the positive DNA diachronic signal and a reduction in molar ellipticity in the negative band (Figure 6C). To determine if BDDE can cleave DNA directly, we incubated BDDE and pBR322 DNA together, and then checked if cleaved fragments were produced. As shown in Figure 6D, no cleaved fragments were observed, even when treated with high concentration of BDDE. These results suggested that there was interaction between DNA and BDDE in vitro; the interaction might include DNA binding as well as intercalation.

Figure 6. Interaction mode between BDDE and DNA. (A) BDDE displaces AO from DNA. AO $(0.5 \mu \mathrm{M})$ was incubated with ctDNA $(50 \mu \mathrm{M})$ in the absence or presence of BDDE at a concentration of $15,30 \mu \mathrm{M}$ for $30 \mathrm{~min}$ at $37{ }^{\circ} \mathrm{C}$, respectively. Fluorescence emission spectra $\left(\lambda_{\text {exc }}=502 \mathrm{~nm}\right)$ were determined using a Hitachi F-4500 fluorescence spectrophotometer (Tokyo, Japan); (B) BDDE displaces Hoechst33258 from DNA. Hoechst33258 $(1.5 \mu \mathrm{M})$ was incubated with ctDNA $(50 \mu \mathrm{M})$ in the absence or presence of BDDE at a concentration of $7.5,15.0 \mu \mathrm{M}$ for $30 \mathrm{~min}$ at $37^{\circ} \mathrm{C}$, respectively. Fluorescence emission spectra $\left(\lambda_{\text {exc }}=352 \mathrm{~nm}\right)$ were analyzed using a Hitachi F-4500 fluorescence spectrophotometer (Tokyo, Japan); (C) Intrinsic CD spectra of ctDNA affected by BDDE. CD spectra of ctDNA alone $(1.5 \mathrm{mM})$ or ctDNA treated with BDDE $(12.5 \mu \mathrm{M})$ were measured with a JASCO 715 spectropolarimeter (Tokyo, Japan); (D) BDDE does not cleave DNA as detected by the agarose gel electrophoresis. Supercoiled plasmid pBR322 DNA was treated without (lane 1) or with 15.5 (lane 2), 31.3 (lane 3), 62.5 (lane 4), 125 (lane 5), 250 (lane 6), 500 (lane 7), and $1000 \mu \mathrm{M}$ (lane 8) BDDE for $30 \mathrm{~min}$ at $37{ }^{\circ} \mathrm{C}$, respectively. The DNA samples were resolved on $1 \%$ agarose, stained with ethidium bromide $(1 \mu \mathrm{g} / \mathrm{mL})$ and photographed under UV light.
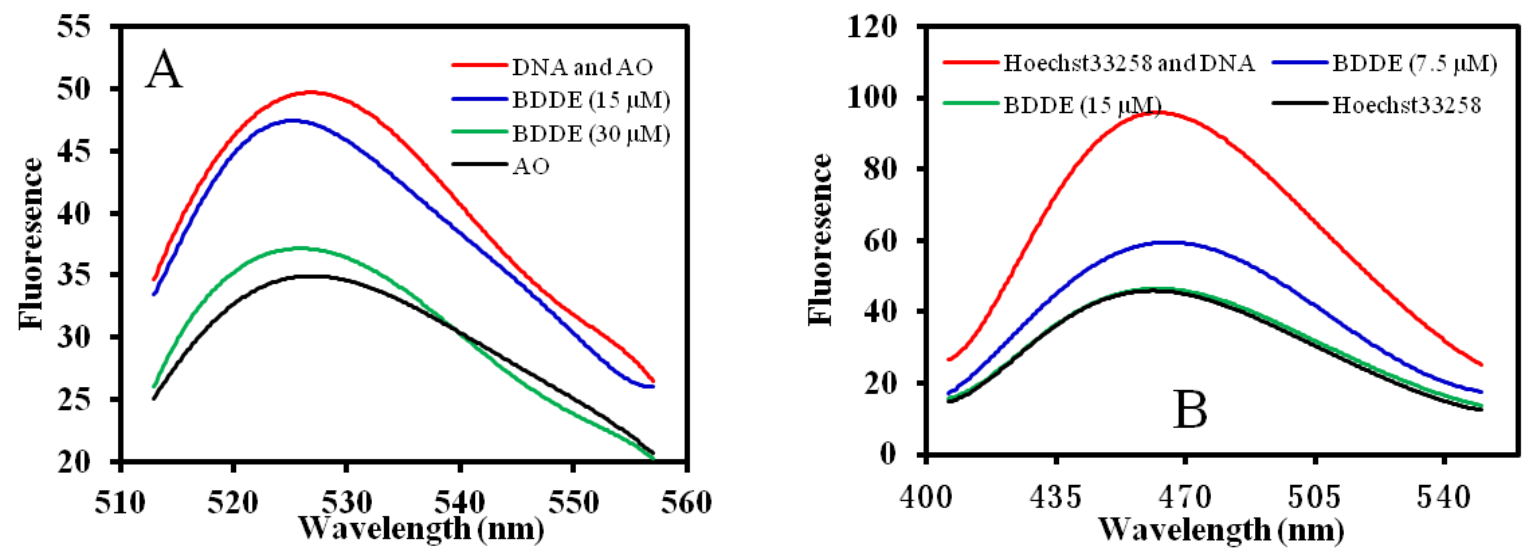
Figure 6. Cont.
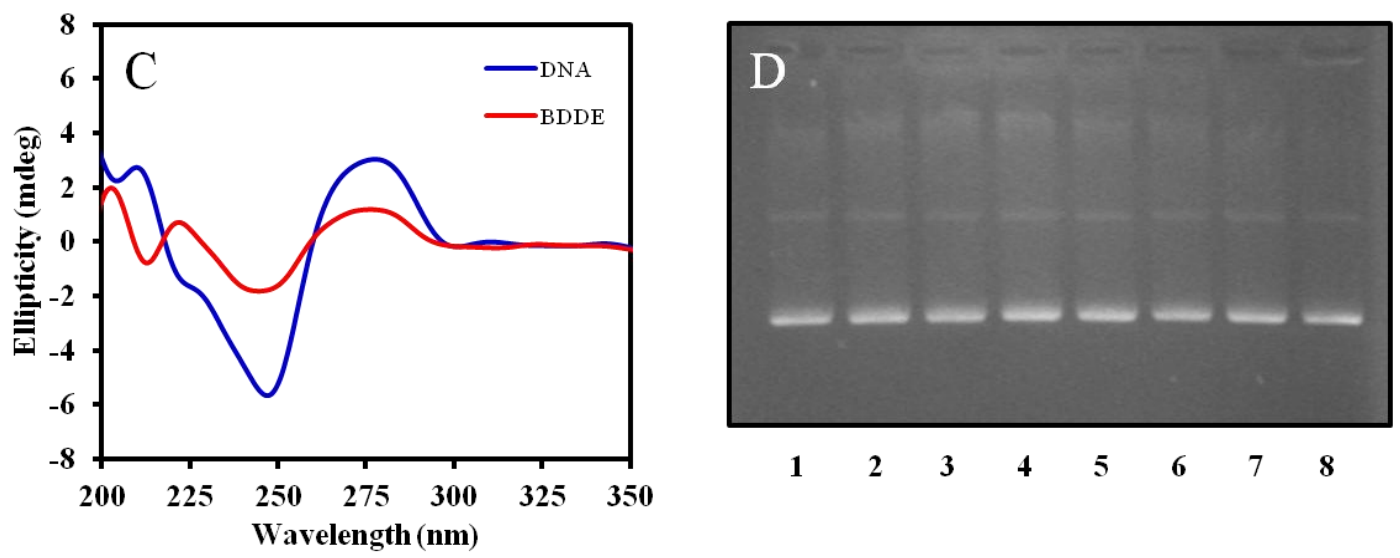

\section{Discussion}

In the present study, we demonstrate that BDDE can inhibit several fungal pathogens, including B. cinerea, Valsa mali, Fusarium graminearum, Coniothyrium diplodiella, and Colletotrichum gloeosporioides. BDDE inhibits the growth of $B$. cinerea most clearly, and prevents the gray mold on strawberries induced by the $B$. cinerea. BDDE can suppress the spore germination and mycelial growth; treatment of the spores with BDDE results in the disruption of the integrity of the cell membrane in $B$. cinerea spores and newly formed germ tubes. Many antifungal agents inhibit the fungal growth by affecting cell membrane permeability. For example, boron decreases gray mold decay by breakdown of the cell membrane and the loss of the cytoplasmic materials [23]; dill oil shows antifungal activities by disrupting the permeability of the plasma membrane [24]. In the present experiments, BDDE induced membrane damage is observed. Loss of membrane integrity in B. cinerea has also been reported in several antifungal agents, including Streptomyces globisporus JK-1 and quinoa (Chenopodium quinoa Willd) alkali. These kinds of compounds usually lead to the leakage of cellular constituents, such as soluble proteins and carbohydrates from hyphae of $B$. cinerea $[25,26]$. Our study provides more evidence that affecting the integrity of the cell membrane is one of the main mechanisms for antifungal agents.

The results from this study reveal that BDDE can increase the cell permeability. However, compared with the inhibition rate $(85 \%)$ on the mycelia growth, the percentage of PI stained spores is very low (23\%), suggesting a lot of dead spores keep membrane integrity and other mechanisms may play some roles in BDDE induced fungal inhibition. Additionally, these two experiments are performed by different approaches. In the analysis of mycelia growth inhibition, the treatment time of BDDE is $2-4$ days. However, in the cell membrane permeabilization experiment, the exposure time of BDDE is only $24 \mathrm{~h}$ before PI staining. Some antifungal agents usually result in mitochondrial dysfunction and intracellular ROS accumulation on fungal pathogens [27]. The effects of BDDE on the mitochondria and ROS need to be addressed further. Also, we have observed that on exposure of strawberries to BDDE for 10 days the antifungal effects are still present (data not shown). These primary results indicated the high stability of the compounds. However, a more systemic study is needed to address if the compound is effective for longer term preservation of strawberries or other fruits. 
In our previous report, we found that BDDE could displace EB from the DNA molecule [13]. In the present study, we show that BDDE can interact with DNA by binding in the minor groove as well as by intercalation; BDDE can also displace DAPI, AO and Hoechst33258. The data provide primary evidence that identification of compounds interacting with DNA molecules is an important strategy for finding novel antifungal agents. However, more studies are needed to address whether BDDE can interact with DNA in vivo; more experiments like co-localization should be performed to document the in vivo interaction between DNA and BDDE.

Currently gray mold on fresh fruits is primarily controlled by application of fungicides in the pre-harvest and sulfur dioxide fumigation in the post-harvest periods, and application of these kinds of compounds usually results in problems of safety in the food supply and in the environment. Marine natural compounds, like BDDE, provide an alternate resource for the development of antifungal agents. Considering the wide antifungal spectrum of BDDE, the compound may be used in the prevention of fungal infections in different plants. Additionally, since marine bromophenols are widely distributed in marine algae and sponges, its application as anticancer, as antioxidant agents or when used as antifungal agent is worthy of further study. However, the safety of application of BDDE in agriculture needs consideration. Our previous study has shown that BDDE possess a relatively low toxicity on a non-tumorigenic epithelial cell line MCF 10A and human vascular endothelium cells (HUVEC) [13]. However, there are reports to show that some bromophenol compounds are suspected of displaying negative impact on human and animal health, acting as endocrine disruptor and moderate toxic agent on mammalian cell [4]. More studies are needed to evaluate the safety of BDDE used as an antifungal agent.

\section{Experimental Section}

\subsection{Drugs, Reagents, and Fruits}

BDDE was synthesized as described previously [18]. Propidium iodide (PI), acridine orange (AO), 4',6-diamidino-2-phenylindole (DAPI), and Hoechst33258 were the products of Beyond, Shanghai, China. Freshly harvested strawberries with no infection or physical injuries were bought from Tianshan orchard, Qingdao, China.

\subsection{Fungal Pathogens}

The fungal pathogens, B. cinerea, Valsa mali, Fusarium graminearum, Coniothyrium diplodiella, Colletotrichum gloeosporioides, Alternaria mali Roberts, and Alternaria porri were kindly provided by College of Chemistry and Pharmaceutical Sciences, Qingdao Agricultural University, Qingdao, China. All of these fungi were cultured on potato dextrose agar (PDA) plates at $23{ }^{\circ} \mathrm{C}$. Spores of B. cinerea were obtained from 14 day old cultures by washing with sterile distilled water containing $0.05 \%(v / v)$ Tween 80 and filtered by sterile cheesecloth. The concentration of the spores was determined using hemocytometer. 


\subsection{Effect of BDDE on Mycelial Growth of Fungal Pathogens on PDA Plates}

The effect of BDDE on mycelial growth of seven fungal pathogens was analyzed in PDA as described previously [28]. Briefly, the fungal pathogens including B. cinerea, Valsa mali, Fusarium graminearum, Coniothyrium diplodiella, Colletotrichum gloeosporioides, Alternaria mali Roberts, and Altenaria porri were cultured on PDA plates. After incubation for 7 days, the mycelial agar (5 mm) was cut and placed in the center of a 6-cm-diameter Petri dish containing PDA without and with $100 \mu \mathrm{g} / \mathrm{mL}$ of BDDE. Radial growth of each fungi was observed after incubation for $2-4$ days at $23{ }^{\circ} \mathrm{C}$ and the inhibition rate of mycelial growth was calculated using the following formula: Inhibition rate of mycelial growth $(\%)=(1-$ diameter of mycelia in the BDDE-treated medium/diameter of mycelia in the no-treatment medium) $\times 100$.

\subsection{Effect of BDDE on Mycelial Growth of B. cinerea in Liquid Medium}

The effect of BDDE on mycelial growth in liquid medium of B. cinerea was analyzed. Briefly, B. cinerea spores were pre-germinated in PDB medium for $24 \mathrm{~h}$ at $23{ }^{\circ} \mathrm{C}$ to form a germ tube. The germ tube and mycelial growth was observed using a microscope after treatment with BDDE $(0-100 \mu \mathrm{g} / \mathrm{mL})$ for another $24 \mathrm{~h}$.

\subsection{Inhibition of Fruit Decay by BDDE}

Effect of BDDE on fruit decay was tested using the methods described previously with little modification [29]. Briefly, freshly harvested healthy strawberries were surface-sterilized in $70 \%$ ethanol for $30 \mathrm{~s}$, and washed with sterile water. All of the fruits were inoculated with a $B$. cinerea spore suspension. Inocula $\left(1.0 \times 10^{6}\right.$ spores $\left./ \mathrm{mL}, 3 \mathrm{~mL}\right)$ were sprayed on about 48 berries with an air-brush sprayer. Berries were dried in air for $1 \mathrm{~h}$, randomized into 4 groups (12/group), and then soaked in certain concentrations of $\operatorname{BDDE}(25,50,100 \mu \mathrm{g} / \mathrm{mL})$ or sterile water for $10 \mathrm{~s}$. After the treatment, fruits were kept in trays covered with plastic film and incubated for 5 days at $23{ }^{\circ} \mathrm{C}$. Damage severity was observed and the decay incidence was calculated using the following formula: Disease incidence $(\%)=($ Number of decayed berries/Total number of treated berries $) \times 100$.

\subsection{Effect of BDDE on Spore Germination and Germ Tube Elongation of B. cinerea}

Inhibition of BDDE on spore germination and germ tube elongation of B. cinerea was measured as described previously with little modifications [30]. In brief, $B$. cinerea spores (final concentration of $5 \times 10^{5}$ spores $/ \mathrm{mL}$ ) were added in PDB medium containing certain concentrations of BDDE $(0-100 \mu \mathrm{g} / \mathrm{mL})$ and incubated at $23{ }^{\circ} \mathrm{C}$ on a rotary shaker at $100 \mathrm{rpm}$ for $12 \mathrm{~h}$. Spores were considered germinated if the germ tube was equal to or greater than the diameter of the spore and a minimum of 100 spores were counted in each replicate under a microscope using a micrometer. The percentage of spore germination was calculated using the following formula: Germinated rate $(\%)=$ (Number of germinated spores/Total number of spores) $\times 100$. 


\subsection{Analysis of Membrane Integrity Using Propidium Iodide (PI) Staining}

Membrane integrity was detected using PI staining as reported previously [23]. Spores of $B$. cinerea were treated with BDDE $(0-100 \mu \mathrm{g} / \mathrm{mL})$ in PDB medium. After incubation at $23{ }^{\circ} \mathrm{C}$ for $24 \mathrm{~h}$, spores were collected by centrifugation and stained with $10 \mu \mathrm{g} / \mathrm{mL}$ propidium iodide (PI) for $10 \mathrm{~min}$ at $30{ }^{\circ} \mathrm{C}$. The spores were washed twice with PBS, and spread onto slides. A minimum of 100 spores were counted under the fluorescence microscopy (Zeiss, Germany). The percentage of PI-stained spores and germ tubes was calculated using the following formula: Percentage of PI negative staining $(\%)=($ Number of PI negative stained spores/Total number of spores $) \times 100$.

\subsection{UV Absorption Spectroscopy}

BDDE $(100 \mu \mathrm{M})$ and DNA $(50 \mu \mathrm{M})$ were dissolved in $50 \mathrm{mM}$ phosphate buffer $(\mathrm{pH} 7.0)$. The absorption spectra were recorded in the presence or absence of BDDE $(100 \mu \mathrm{M})$ using a Beckman DU 650 UV-VIS spectrometer (Kleve, Germany), respectively.

\subsection{Circular Dichroism Spectroscopy for Secondary Structure of ctDNA}

CD spectra $(220-300 \mathrm{~nm})$ of ctDNA $(1.5 \mathrm{mM})$ treated with BDDE $(12.5 \mu \mathrm{M})$ were measured with a JASCO 715 spectropolarimeter (JASCO, Tokyo, Japan). The spectra were collected and corrected by reduction of noise and smoothing using the program JWSSE (JASCO, Tokyo, Japan).

\subsection{DAPI, AO, and Hoechst33258 Displacement Fluorescence Assay}

DAPI, AO, and Hoechst33258 displacement fluorescence assay were employed to determine the interaction and interaction mode between BDDE and DNA. ctDNA $(50 \mu \mathrm{M})$ was dissolved in $50 \mathrm{mM}$ phosphate buffer $(\mathrm{pH} 7.0)$ and incubated with DAPI $(1.5 \mu \mathrm{M})$, AO $(0.5 \mu \mathrm{M})$, and Hoechst33258 $(1.5 \mu \mathrm{M})$, respectively. Certain concentrations of BDDE were added and incubated for $30 \mathrm{~min}$ at $37{ }^{\circ} \mathrm{C}$. Fluorescence emission spectra of DAPI, AO, and Hoechst33258 were determined using a Hitachi F-4500 fluorescence spectrophotometer (JASCO, Tokyo, Japan).

\subsection{Agarose Gel Electrophoresis}

Supercoiled plasmid pBR322 DNA $(0.25 \mu \mathrm{g})$ was dissolved in the reaction buffer (35 $\mathrm{mM}$ Tris- $\mathrm{HCl}, 72 \mathrm{mM} \mathrm{KCl}, 5 \mathrm{mM} \mathrm{MgCl}_{2}, 5 \mathrm{mM}$ DTT, $5 \mathrm{mM}$ spermidine and $0.1 \% \mathrm{BSA}$ ). Certain concentrations of BDDE $(0-1000 \mu \mathrm{M})$ were added in the reaction buffer and incubated for $30 \mathrm{~min}$ at $37{ }^{\circ} \mathrm{C}$. The reaction was terminated by lyophilizing at $-80{ }^{\circ} \mathrm{C}$ and DNA samples were resolved on $1 \%$ agarose, stained with ethidium bromide $(1 \mu \mathrm{g} / \mathrm{mL})$ and photographed under UV light.

\subsection{Data Analysis}

Student's $t$-test was used for statistical analysis of the data, and values were expressed as mean \pm SD. Differences of $p<0.05$ were considered statistically significant. 


\section{Conclusions}

In conclusion, the present study reveals that the bromophenol BDDE displays inhibition against several fungal pathogens. BDDE decreases the germination rate, inhibits the mycelial growth of B. cinerea, and destroys the integrity of the fungal cell membrane. BDDE interacts with DNA via intercalation and minor groove binding, and both the membrane disruption effect and the DNA-binding activities may contribute to the antifungal effects in BDDE-induced inhibition of fungal pathogens. With the unique chemical structure different from the current fungicides, BDDE may serve as a novel antifungal candidate or parent compound for rational drug design.

\section{Acknowledgments}

This work was supported by the National Natural Science Foundation of China (No. 81001396 and No. 81273550) and State Innovative Drugs Development Program of China (No. 2013ZX09103003019). We are also grateful to Fred Bogott at the Minnesota University and Poul Erik Hansen in Roskilde University for their careful editing of the manuscript.

\section{Author Contributions}

Ming Liu, Lin Xiao, and Xiukun Lin contributed to the study concept and design, and the manuscript preparation. Ming Liu, Lin Xiao and Genzhu Wang performed the experimental studies and analyzed the data. Xuanli $\mathrm{Xu}$, Xiaohui Liu, and Pingxiang Xu helped acquire data and statistical analysis. Ming Liu, Genzhu Wang., and Xiukun Lin revised the article critically for intellectual content.

\section{Conflicts of Interest}

The authors declare no conflict of interest.

\section{References}

1. Gonzalez-Fernandez, R.; Jorrin-Novo, J.V. Contribution of proteomics to the study of plant pathogenic fungi. J. Proteome Res. 2012, 11, 3-16.

2. Elad, Y.; Gullino, M.L.; Shtienberg, D.; Aloi, C. Managing Botrytis cinerea on tomatoes in greenhouses in the Mediterranean. Crop Prot. 1995, 14, 105-109.

3. Soylu, E.M.; Kurt, SS.; Soylu, S. In vitro and in vivo antifungal activities of the essential oils of various plants against tomato grey mould disease agent Botrytis cinerea. Int. J. Food Microbiol. 2010, 143, 183-189.

4. Liu, M.; Hansen, P.E.; Lin, X. Bromophenols in marine algae and their bioactivities. Mar. Drugs 2011, 9, 1273-1292.

5. Popplewell, W.L.; Northcote, P.T. Colensolide A: A new nitrogenous bromophenol from the New Zealand marine red alga Osmundaria colensoi. Tetrahedron Lett. 2009, 50, 6814-6817.

6. Xu, N.; Fan, X.; Yan, X.; Li, X.; Niu, R.; Tseng, C.K. Antibacterial bromophenols from the marine red alga Rhodomela confervoides. Phytochemistry 2003, 62, 1221-1224. 
7. Chen, M.; Shao, C.L.; Fu, X.M.; Xu, R.F.; Zheng, J.J.; Zhao, D.L.; She, Z.G.; Wang, C.Y. Bioactive indole alkaloids and phenyl ether derivatives from a marine-derived Aspergillus sp. Fungus. J. Nat. Prod. 2013, 76, 547-553.

8. Park, H.J.; Kurokawa, M.; Shiraki, K.; Nakamura, N.; Choi, J.S.; Hattori, M. Antiviral activity of the marine alga Symphyocladia latiuscula against herpes simplex virus (HSV-1) in vitro and its therapeutic efficacy against HSV-1 infection in mice. Biol. Pharm. Bull. 2005, 28, 2258-2262.

9. Kim, S.Y.; Kim, S.; Oh, M.J.; Jung, S.J.; Kang, S. In Vitro antiviral activity of red alga, Polysiphonia morrowii extract and its bromophenols against fish pathogenic infectious hematopoietic necrosis virus and infectious pancreatic necrosis virus. J. Microbiol. 2011, 49, 102-106.

10. Xu, X.; Piggott, A.M.; Yin, L.; Capon, R.J.; Song, F. Symphyocladins A-G: Bromophenol adducts from a Chinese marine red alga, Symphyocladia latiuscula. Tetrahedron Lett. 2012, 53, 2103-2106.

11. Lee, H.S.; Lee, T.H.; Lee, J.H.; Chae, C.S.; Chung, S.C.; Shin, D.S.; Shin, J.; Oh, K.B. Inhibition of the pathogenicity of magnaporthe grisea by bromophenols, isocitrate lyase inhibitors, from the red alga Odonthalia corymbifera. J. Agric. Food Chem. 2007, 55, 6923-6928.

12. Xu, X.; Song, F.; Wang, S.; Li, S.; Xiao, F.; Zhao, J.; Yang, Y.; Shang, S.; Yang, L.; Shi, J. Dibenzyl bromophenols with diverse dimerization patterns from the brown alga Leathesia nana. J. Nat. Prod. 2004, 67, 1661-1666.

13. Liu, M.; Zhang, W.; Wei, J.; Qiu, L.; Lin, X. Marine bromophenol bis(2,3-dibromo-4, 5-dihydroxybenzyl) ether, induces mitochondrial apoptosis in K562 cells and inhibits topoisomerase I in vitro. Toxicol. Lett. 2012, 211, 126-134.

14. Shi, D.; Xu, F.; He, J.; Li, J.; Fan, X.; Han, L. Inhibition of bromophenols against PTP1B and anti-hyperglycemic effect of Rhodomela confervoides extract in diabetic rats. Chin. Sci. Bull. 2008, 53, 2476-2479.

15. Kurihara, H.; Mitani, T.; Kawabata, J.; Takahashi, K. Inhibitory potencies of bromophenols from Rhodomelaceae algae against $\alpha$-glucosidase activity. Fish Sci. 1999, 65, 300-303.

16. Kurihara, H.; Mitani, T.; Kawabata, J.; Takahashi, K. Two new bromophenols from the red alga Odonthalia corymbifera. J. Nat. Prod. 1999, 62, 882-884.

17. Kim, K.Y.; Nguyen, T.H.; Kurihara, H.; Kim, S.M. Alpha-glucosidase inhibitory activity of bromophenol purified from the red alga Polyopes lancifolia. J. Food Sci. 2010, 75, H145-H150.

18. Liu, M.; Zhang, W.; Wei, J.; Lin, X. Synthesis and $\alpha$-glucosidase inhibitory mechanisms of bis(2,3-dibromo-4,5-dihydroxybenzyl) ether, a potential marine bromophenol $\alpha$-glucosidase inhibitor. Mar. Drugs 2011, 9, 1554-1565.

19. Lou, L.; Velligan, M.; Roberts, C.; Stevens, D.A.; Clemons, K.V. DNA binding compounds targeting fungal pathogens: An emerging concept in the discovery of novel antifungal agents. Curr. Opin. Investig. Drugs 2002, 3, 1437-1445.

20. Banerjee, D.; Pal, S.K. Dynamics in the DNA recognition by DAPI: Exploration of the various binding modes. J. Phys. Chem. B 2008, 112, 1016-1021.

21. Nafisi, S.; Saboury, A.A.; Keramat, N.; Neault, J.F.; Tajmir-Riahi, H.A. Stability and structural features of DNA intercalation with ethidium bromide, acridine orange and methylene blue. J. Mol. Struct. 2007, 827, 35-43. 
22. Palchaudhuri, R.; Hergenrother, P.J. DNA as a target for anticancer compounds: methods to determine the mode of binding and the mechanism of action. Curr. Opin. Biotechnol. 2007, 18, 497-503.

23. Qin, G.; Zong, Y.; Chen, Q.; Hua, D.; Tian, S. Inhibitory effect of boron against Botrytis cinerea on table grapes and its possible mechanisms of action. Int. J. Food Microbiol. 2010, 138, $145-150$.

24. Tian, J.; Ban, X.; Zeng, H.; He, J.; Chen, Y.; Wang, Y. The mechanism of antifungal action of essential oil from dill on Aspergillus flavus. PLoS One 2012, 7, e30147.

25. Li, Q.; Ning, P.; Zheng, L.; Huang, J.; Li, G.; Hsiang, T. Effects of volatile substances of Streptomyces globisporus JK-1 on control of Botrytis cinerea on tomato fruit. Biol. Control 2012, $61,113-120$.

26. Stuardo, M.; San Martín, R. Antifungal properties of quinoa (Chenopodium quinoa Willd) alkali treated saponins against Botrytis cinerea. Ind. Crop. Prod. 2008, 27, 296-302.

27. Helmerhorst, E.J.; Troxler, R.F.; Oppenheim, F.G. The human salivary peptide histatin 5 exerts its antifungal activity through the formation of reactive oxygen species. Proc. Natl. Acad. Sci. USA 2001, 98, 14637-14642.

28. Droby, S.; Wisniewski, M.; El Ghaouth, A.; Wilson, C. Influence of food additives on the control of postharvest rots of apple and peach and efficacy of the yeast-based biocontrol product aspire. Postharvest Biol. Technol. 2003, 27, 127-135.

29. Huang, R.; Che, H.J.; Zhang, J.; Yang, L.; Jiang, D.H.; Li, G.Q. Evaluation of Sporidiobolus pararoseus strain YCXT3 as biocontrol agent of Botrytis cinerea on post-harvest strawberry fruits. Biol. Control 2012, 62, 53-63.

30. Qin, G.Z.; Tian, S.P.; Xu, Y.; Wan, Y.K. Enhancement of biocontrol efficacy of antagonistic yeasts by salicylic acid in sweet cherry fruit. Physiol. Mol. Plant Pathol. 2003, 62, 147-154.

(C) 2014 by the authors; licensee MDPI, Basel, Switzerland. This article is an open access article distributed under the terms and conditions of the Creative Commons Attribution license (http://creativecommons.org/licenses/by/3.0/). 